\title{
Newtonian dynamics of imaginary time-dependent mean field theory
}

\author{
Aldo Bonasera \\ Cyclotron Institute, Texas A\&M University, College Station, TX 77843-USA. \\ Laboratori Nazionali del Sud-INFN, v. Santa Sofia 64, 95123 Catania, Italy.
}

\begin{abstract}
A Time Dependent Hartree-Fock (TDHF) based classical model is applied to sub-barrier fusion reactions using the Feynman Path Integral Method (FPIM). The fusion cross-sections and modified astrophysical $\mathrm{S}^{*}$-factors are calculated for the ${ }^{12} \mathrm{C}+{ }^{12} \mathrm{C}$ reactions and compared to direct and indirect experimental results. Different channels cross-sections are estimated from the statistical decay of the compound nucleus. A good agreement with the direct data is found. We suggest a complementary observable given by the (imaginary) action A easily derived from theory and experiments. When properly normalized by the action in the Gamow limit it has an upper value of 1 at zero beam energies. It becomes negative at the Coulomb barrier which is $\mathrm{V}_{\mathrm{cb}}=5.05 \pm 0.05 \mathrm{MeV}$ from direct data and $\mathrm{V}_{\mathrm{cb}}=5.5 \mathrm{MeV}$ from model calculations.
\end{abstract}

Careful measurements of the fusion cross-sections for ${ }^{12} \mathrm{C}+{ }^{12} \mathrm{C}$ reactions are crucial to our understanding of massive stars and super-bursts from accreting neutron stars [1-4]. These measurements are especially compelling at energies close or below the Gamow peak [4], i.e., below $2 \mathrm{MeV}$. Direct data measurements [5-10] start from $\mathrm{E}_{\mathrm{cm}}=2.1 \mathrm{MeV}$ center of mass energy showing many resonances especially when expressed in terms of the modified astrophysical $\mathrm{S}^{*}$ factor [13]:

$$
\begin{gathered}
\mathrm{S}^{*}\left(\mathrm{E}_{\mathrm{cm}}\right)=\mathrm{E}_{\mathrm{cm}} \sigma\left(\mathrm{E}_{\mathrm{cm}}\right) \times \exp \left(87.12 \mathrm{E}_{\mathrm{cm}}{ }^{-1 / 2}+0.46 \mathrm{E}_{\mathrm{cm}}\right)= \\
\mathrm{S}\left(\mathrm{E}_{\mathrm{cm}}\right) \times \exp \left(0.46 \mathrm{E}_{\mathrm{cm}}\right) .
\end{gathered}
$$

To overcome the difficulties of vanishing fusion crosssection at very low energies, indirect methods have been proposed [14,15]. Recently, indirect measurements based on the THM have been published [11] displaying a wealth of resonances in $\mathrm{S}^{*}$ at center of mass energies as low as $0.8 \mathrm{MeV}$. A critical reanalysis of the same experiment to include Coulomb effects in the 3-body final channel and the Distorted Wave Born Approximation (DWBA) instead of the Plane Wave Approximation (PWA) resulted in a much lower $\mathrm{S}^{*}$ up to three orders of magnitude. These contrasting results reveal a strong model dependence of the THM approach and cast doubts about its general validity. Macroscopic and microscopic models [16-19] offer a reasonable reproduction of the direct data and they are sometime considered as an upper limit of the fusion cross-section [20,21]. Predictions of these models cannot reproduce the low energy THM results of ref.[11] and are way above the Coulomb renormalization analysis of [12].

In ref. [22], we proposed a microscopic model based on the Vlasov equation and the FPIM reproducing rather well the available direct data at that time for the ${ }^{12} \mathrm{C}+{ }^{12} \mathrm{C}$ system. The approach is quite successful when applied to heavier nuclei [23] and spontaneous fission [24]. Before getting involved into complex numerical calculations, which may or may not be feasible at such low energies, it is instructive to simplify the heavy ion dynamics using a robust macroscopic model. In ref. [25], we proposed a TDHF based model, dubbed Neck Model (NM), and successfully reproduced above the barrier fusion cross section as well as deep inelastic [26] and fission [27] dynamics. In this work we generalize the NM to sub barrier energies in the FPIM framework. Newton force equations are solved assuming the collective variables given by the center of mass distance $\mathbf{R}$ of the two colliding nuclei and their relative momenta $\mathbf{P}$. The forces acting on the nuclei are given by the Coulomb and the nuclear Bass potentials before the two nuclei touch [28]. After touching, the two nuclei are described as sections of spheres joined by a cylinder of radius $r_{N}$, the neck radius. In the rebounding phase, the nuclear geometry is given by two half spheres joined by sections of cones of radius $r_{N}$. Volume conservation is enforced which gives suitable relations between the neck radius and the relative distance of the two nuclei and it is in good agreement to TDHF calculations [25]. In such a configuration the force is given by the surface tension times the perimeter of the neck, as for a liquid drop. During this stage nucleons are transferred through the neck, giving rise to one body-dissipation described by the Randrup-window formula [29]. To describe the subbarrier fusion dynamics, we go into imaginary times (FPIM) at the first (external) turning point [22-24] when the collective momentum $\mathrm{P}=0 \mathrm{fm}^{-1}$. In imaginary times, the second derivative of $\mathrm{R}$ respect to (imaginary) time gets a $i^{2}=-1$ contribution and the Coulomb force becomes attractive while the nuclear part repulsive. This produces an acceleration of the two nuclei towards each other until they come to a halt (because of the short-range nuclear 
repulsion in imaginary times) at the second (internal) turning point. At this stage we switch from imaginary to real times again. If the nuclear force is strong enough the two nuclei fuse and this happens always for light nuclei like ${ }^{12} \mathrm{C}$. The probability of fusion for the lth-partial wave is given by [22]:

$$
T_{l}=(1+\exp \{2 A\})^{-1}
$$

The action (in units of $\hbar$ ) is given by

$$
A=\int_{1}^{2} P d R \text {. }
$$

The cross section is given by:

$$
\sigma\left(E_{c m}\right)=\frac{\pi \hbar^{2}}{2 \mu E_{c m}} \sum_{l=0}^{\infty}(2 l+1) T_{l} P_{l}
$$

$\mathrm{P}_{1}$ gives the probability of decay of the compound nucleus into different channels, is the nuclear reduced mass. Following [22], the calculations will be performed at zero impact parameter only. In order to take into account the 1-dependence of the transmission probability we shift the beam energy for each 1 as [30]:

$$
T_{l} \approx T_{0}\left(E_{c m}-\frac{l(l+1) \hbar^{2}}{2 \mu<R^{2}>}\right)
$$

$\mu<\mathrm{R}^{2}>$ is an effective moment of inertia of two touching spheres at the internal turning point $\angle \mathrm{R}>$ and it is slowly varying with energy. Recall that for the ${ }^{12} \mathrm{C}+{ }^{12} \mathrm{C}$ system, because of angular momentum and parity conservation laws, only even l-values are allowed.

In figure 1, we plot, for different 1-values as indicated, the collective momentum versus collective distance of the two nuclei at $\mathrm{E}_{\mathrm{cm}}=2.5 \mathrm{MeV}$. Starting from infinite distance, the two nuclei approach each other under the influence of the Coulomb repulsion until they reach the first turning point $\left(\mathrm{P}=0 \mathrm{fm}^{-1}\right)$. Clearly the first turning point is farther for higher 1-values or lower beam energies. At this stage, the system enters imaginary time propagation, and the Coulomb force becomes attractive resulting in a strong acceleration. At shorter distances the (repulsive in imaginary times) nuclear force given by the Bass potential violently decelerates the two nuclei until $\mathrm{P}=0 \mathrm{fm}^{-1}$ again, the second turning point. For light nuclei the imaginary dynamics is completely determined by the Coulomb and Bass potential. When returning to real times, the two nuclei accelerate again towards each other, a neck is formed, and fusion occurs. The area inside the first and second turning points gives the action A. Notice that A increases quickly with increasing 1 , thus the probability of fusion decreases, eq.(1), and the cross section is dominated by low 1 values, eq.(2). A striking difference with the microscopic Vlasov approach, see figure 2 in ref.[22], is the oscillations seen in imaginary times. These are due to collective excitation of the single particle degrees of freedom included in the microscopic model but not in the NM. As a result, oscillations are seen in the fusion cross section of figure 3 in ref.[22] and we will discuss them further in the fig.3 below.

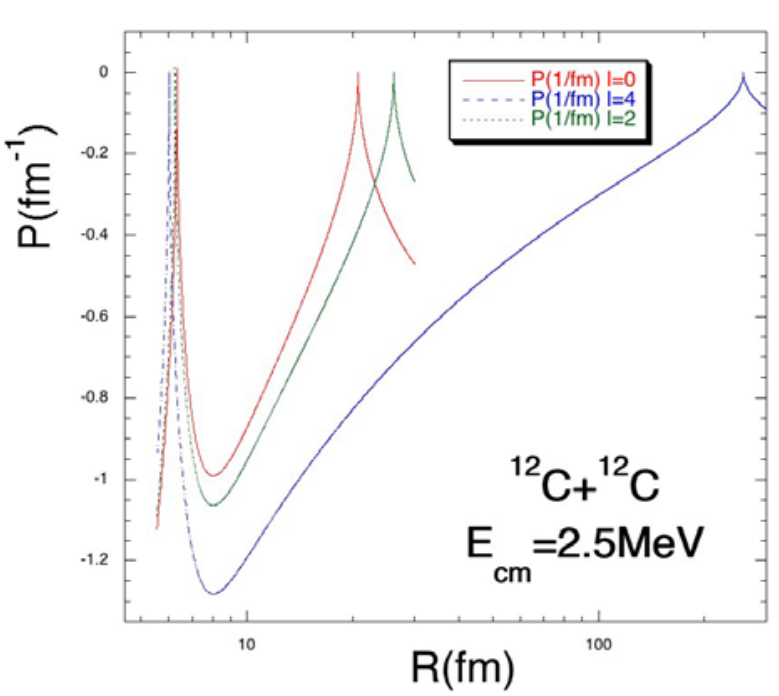

Figure 1. Collective momentum vs distance of the two nuclei for the values of $\mathrm{E}_{\mathrm{cm}}$ and 1 indicated. The areas inside the first and second turning points give the action A.

After fusing, the excitation energy of the compound nucleus $(\mathrm{CN})$ can be determined from the $\mathrm{E}_{\mathrm{cm}}, 1$ and the $\mathrm{Q}$-values for the $\mathrm{CN}$ and the open decay channels. In our case the most important channels are: ${ }^{12} \mathrm{C}\left({ }^{12} \mathrm{C}, \gamma\right){ }^{24} \mathrm{Mg}, \mathrm{Q}=13.9 \mathrm{MeV} ;{ }^{12} \mathrm{C}\left({ }^{12} \mathrm{C}, \alpha\right)$ Ne $\mathrm{Q}=4.62$ $\mathrm{MeV} ;{ }^{12} \mathrm{C}\left({ }^{12} \mathrm{C}, \mathrm{p}\right){ }^{23} \mathrm{Na}, \mathrm{Q}=2.24 \mathrm{MeV} ;{ }^{12} \mathrm{C}\left({ }^{12} \mathrm{C}, \mathrm{n}\right){ }^{23} \mathrm{Mg}$, $\mathrm{Q}=-2.6 \mathrm{MeV} ;{ }^{12} \mathrm{C}\left({ }^{12} \mathrm{C},{ }^{8} \mathrm{Be}\right){ }^{16} \mathrm{O}, \mathrm{Q}=-0.2 \mathrm{MeV}$.

We can calculate the decay probability in certain channels within the framework of the statistical model [32]:

$$
\Pi_{i}=g_{i}\left(T_{k_{i}}^{2}+2 m_{i} T_{k_{i}}\right) \frac{\rho_{i}\left(E_{C N}^{*}-T_{k_{i}}\right)}{\rho\left(E_{C N}^{*}\right)} \sigma_{i}^{a b s}
$$

The probability entering eq. (2) can be expressed as $P_{l}=\Pi_{i} / \sum \Pi_{i}$ for each 1-value and excitation energy

$$
\begin{aligned}
& E_{C N}^{*}=E_{c m}-\frac{l(l+1) \hbar^{2}}{2 \mu<R^{2}>}+Q . \\
& g_{i}=2 s_{i}+1
\end{aligned}
$$

and $\mathrm{s}_{\mathrm{i}}$ is the spin of the emitted particles. The absorption cross section for the inverse process is given by:

$\sigma_{i}^{a b s}=\pi R_{N}^{2}\left(1-V_{C}\left(R_{N}\right) / T_{k_{i}}\right)$, where $\mathrm{R}_{\mathrm{N}}$ is the sum of the radii of the nuclei in the exit channel, $V_{C}\left(R_{N}\right)$ the Coulomb barrier and $\mathrm{T}_{\mathrm{ki}}$ the kinetic energy of the emitted particle. For gamma absorption:

$$
\sigma_{\gamma}^{a b s}=75 A_{C N} \frac{\left(T_{k_{\gamma}}-2.226\right)^{\frac{3}{2}}}{T_{k \gamma}^{3}}(\mathrm{mb})
$$

$\mathrm{A}_{\mathrm{CN}}$ the mass number of the $\mathrm{CN}$. The level densities contribution can be written as:

$$
\frac{\rho_{i}\left(E_{C N}^{*}-T_{k_{i}}\right)}{\rho\left(E_{C N}^{*}\right)}=\exp \left(-\frac{T_{k_{i}}}{T}\right) ;
$$

the temperature is given by

$$
T=\sqrt{E_{C N}^{*} / a} \text { and } \mathrm{a}=\mathrm{A}_{\mathrm{CN}} / 8.0\left(\mathrm{MeV}^{-1}\right) .
$$




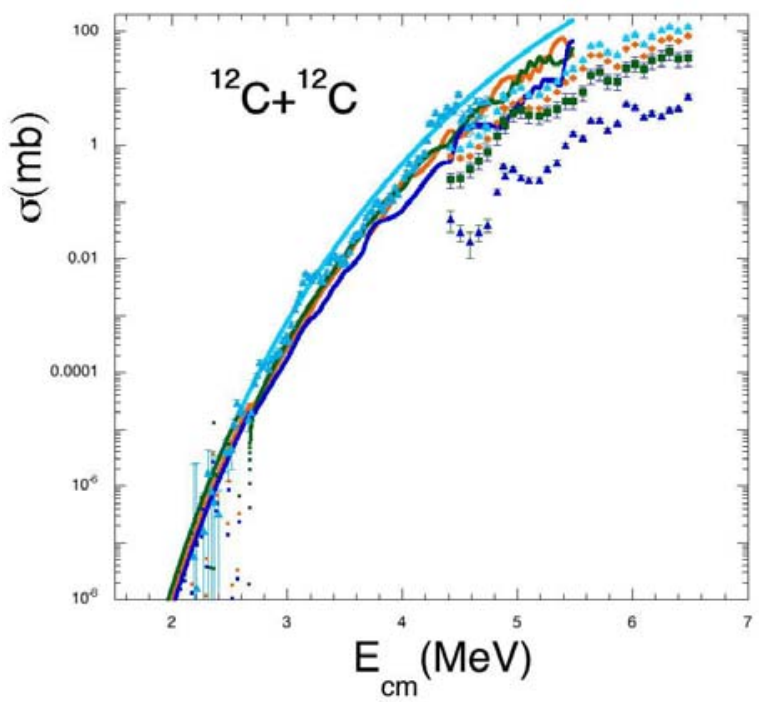

Figure 2. Fusion cross-section as function of $E_{c m}$ (open symbols) compared to the direct data (full symbols) $[7,8]$. The total cross section is given by the triangles (cyan), -channel by the diamonds (orange), $\mathrm{n}$-channel by the circles (green) and p-channel (blue) by the squares. The model agrees to the data from ref.[8] and it overestimate ref.[7]. Notice the faster increase of the n-channel (negative Q-value) compared to the p-channel. The model reproduces the trend but overestimates the data of ref.[7].

In figure 2, we compare the model results to the direct data $[7,8]$ for the total cross section and the most important decay channels ( $\alpha, p$ and $n)$. The overall agreement is quite good especially at the highest energies. An over (under) estimate of the $n(p)$-channel is noticeable. At lower excitation energies the individual levels of the $\mathrm{CN}$ and the exit channels nuclei become important for each l-values, these levels are not included in the present calculations, and we expect them to produce oscillations as in the data if properly considered. Notice that any statistical model refinement will affect the $\mathrm{P}_{1}$ values entering eq. (1) but such a quantity is below one, thus we conclude that the calculated cross-sections represent an upper limit [20,21]. Our results are in good agreement with other model calculations [16-20].

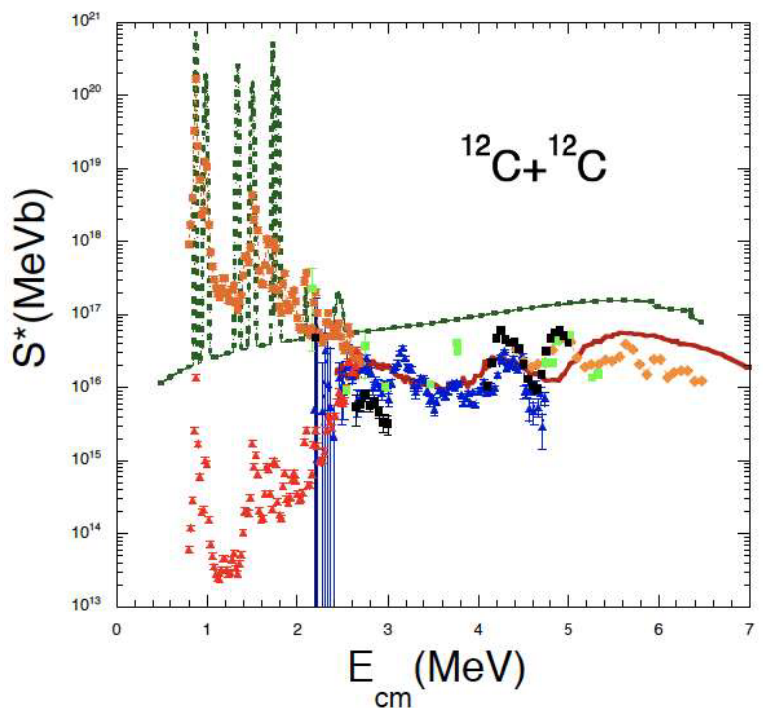

Figure 3. Modified Astrophysical S*-factor as function of $\mathrm{E}_{\mathrm{cm}}$. Direct data are from $[7,8,33,34]$. The THM results are given by the full (orange) circles [11] and the Coulomb renormalized one by the full (red) triangles [12]. The neckmodel calculations, including ${ }^{24} \mathrm{Mg}$ resonances, are given by the dashed (green) line [35]. The Vlasov results in imaginary times are given by the full (red) line [22].

To avoid the quickly decreasing fusion cross-section at sub-barrier energies, it is customary to display the modified astrophysical factor $\mathrm{S}^{*}$ as function of $\mathrm{E}_{\mathrm{cm}}$, see figure 3. The model calculations give $\mathrm{S}^{*}=1.310^{16} \mathrm{MeV}$ $\mathrm{b}$ at the lowest energy, slowly increasing over the entire energy region. Direct data $[7,8]$ are overestimated by the calculation at higher energies and display definite 'bumps'. The indirect data are normalized to the direct data at $2.6 \mathrm{MeV}$ [11]. At much lower energies, the THM diverges from the calculation up to 3 orders of magnitude higher, while the Coulomb renormalized THM analysis is up to 3 orders below the calculation. These discrepancies need to be experimentally investigated maybe within the same THM approach but for a system, which does not present the same Coulomb problem for one of the outgoing particles. A suitable reaction could be ${ }^{13} \mathrm{C}\left({ }^{12} \mathrm{C}, \mathrm{n}\right)$... where the neutron may act as a spectator [12]. In ref. [35] we have extended the present model to include effects of known resonances in ${ }^{26} \mathrm{Mg}$. We found a reasonable agreement to the THM data [11]. In ref. [36], we have added $\mathrm{e}^{+} \mathrm{e}^{-}$corrections to the Coulomb field resulting in a $15 \%$ decrease in $\mathrm{S}^{*}$, closer to the direct data. The Vlasov calculations [22] are in rather good agreement to the direct data and display oscillations or 'bumps' similar to the data. A repetition of those calculations extending to lower energies and different interactions would be very interesting.

Calculated and measured values of $\mathrm{S}$ or $\mathrm{S}^{*}$ vary widely from system to system and as a function of $E_{c m}$ [31]. The original purpose in defining these observables was to avoid the large variation of the fusion cross-section by factoring out the Coulomb barrier penetration probability [4] thus leaving nuclear effects embedded into S or S*. Whether this goal has been reached can be judged from figure 3 and the literature [1-13,16-21,31]. In this work we would like to propose a complementary observable which can be derived from eq. (2). We have shown in figure 1 that at the lowest energies the $l=0$ value is dominant, thus neglecting higher l-values and solving for $\mathrm{T}_{0}$, the action becomes:

$$
A=\frac{1}{2} \ln \left[\frac{\pi \hbar^{2}}{2 E_{c m} \sigma\left(E_{c m}\right)}-1\right]
$$

Of course, if data could be provided for a particular 1value, then eq.(4) could be modified to correct for it, see eq.(2). Notice that when dealing with the total fusion cross-section $\mathrm{P}_{1}=1$. The value of the action has been derived analytically for the case of the Coulomb barrier only (Gamow limit) [31]

$$
A_{G}=e^{2} \pi Z_{T} Z_{P} \sqrt{\frac{\mu}{2 E_{C M}}} .
$$

Thus, we expect $A \rightarrow A_{G}$ when $E_{C M} \rightarrow 0$. 


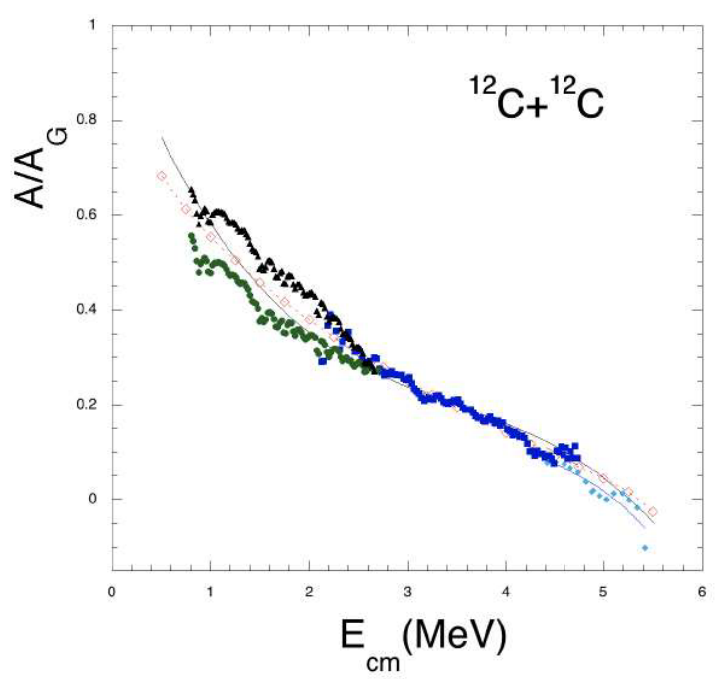

Figure 4. Normalized sub barrier action, symbols as in figure 3. The dashed line is the fit to the model using eq.(5), the full line is the fit to the direct data of ref.[7] and the dashed line to ref.[8].

In figure 4, we plot the Gamow normalized action vs $\mathrm{E}_{\mathrm{cm}}$ for the model not including resonances [35] (open symbols) and the data (full symbols). We notice a reasonable agreement to the direct data, while the THM is below [11] or above the calculations for the Coulomb renormalized case [12], i.e., similar to the figure 3 . Notice that the action A has an opposite behavior to $\mathrm{S}$ and $\mathrm{S}^{*}$, i.e., the higher is $\mathrm{A}$ the smaller is the cross section. Because of the clear physical interpretation of $\mathrm{A} / \mathrm{A}_{\mathrm{G}}$ we can parameterize its behavior as:

$$
A / A_{G}=a+b e^{-c E_{c m}}
$$

The parameters $a, b, c$ is fitted to data or theory and the results can be seen in figure 3 . The action becomes zero at the effective Coulomb barrier $\mathrm{V}_{\mathrm{cb}}=5.05 \pm 0.05 \mathrm{MeV}$ from the direct data $[7,8]$ to compare to the model $\mathrm{V}_{\mathrm{cb}}=5.5 \mathrm{MeV}$. The latter is the highest energy we run the simulation and its value critically depends on the Bass or other nuclear potentials. The fits to the indirect data give $\mathrm{V}_{\mathrm{cb}}=4.3 \mathrm{MeV}$ for the Coulomb renormalization case [12] and no solution for the original data [11]. Of course, this last result depends on the THM normalization [11] to the direct data [8]. There seem to be some discrepancy between the direct data of ref. [7] and [8] with the former better reproduced by the model.

In conclusion, in this paper we have discussed a TDHF inspired macroscopic model and extended it to subbarrier energies using the Feynman Path Integral Method i.e., solving Newton dynamics below the barrier in imaginary times. The model has no free parameters and reasonably reproduces the direct data. The THM indirect data are well above or below the model depending on if the Coulomb corrections are applied [12] or not [11]. Inclusion of resonances in the model [35] supports the results of ref. [11]. Coulomb field modifications due to $\mathrm{e}+\mathrm{e}-$ corrections gives results in better agreement to the direct data [36]. We have introduced a new observable, the action $\mathrm{A}$, that when properly normalized by the action in the Gamow limit gives a quantity well defined and close to unity and we expect all the other systems to fall in the same region.

\section{ACKNOWLEDGEMENTS}

We thank Profs. J. Natowitz, A. Tumino and A. Zhanov for discussions and providing the THM data. In particular, we thank prof. A.Tumino for help to recover the results from the imaginary time Vlasov calculations [22] displayed in fig.3. This work was supported in part by the United States Department of Energy under Grant \# DE-FG03-93ER40773 and NNSA Grant No. DENA0003841 (CENTAUR).

\section{REFERENCES}

[1] Woosley, S. E., Heger, A. and Weaver, T. A. Rev. Mod. Phys. 74,1015-1071 (2002).

[2] Keek L.et al., Atron.Astrophys.,479,177(2008).

[3] Cooper, R. L. et al., Astrophys.J. 702, 660-671 (2009).

[4] Iliadis, C. Nuclear Physics of Stars. (Wiley,

Weinheim, 2007).

[5] H.W. Becker et al., Z. Phys. A 303, 305 (1981).

[6] K.U. Kettner, H. Lorenz-Wirzba, C. Rolfs, Z. Phys. A 298, 65(1980).

[7] E.F. Aguilera et al., Phys. Rev. C 73, 064601 (2006).

[8] T. Spillane et al., Phys. Rev. Lett. 98, 122501 (2007).

[9] J.R. Patterson, H. Winkler, C.S. Zaidins, Astrophys. J. 157, 367(1969).

[10] C.L. Jiang et al., Phys. Rev. C 97, 012801(R) (2018).

[11] A. Tumino et al., Nature 557, 687 (2018).

[12] C.Beck et al., Eur. Phys. J. A56, 87 (2020).

[13] Patterson, J. R., Winkler, H. \& Zaidins, C. S., Astrophys. J. 157, 367-373(1969).

[14] Tribble, R. et al.,Rep. Prog.Phys. 77, 106901106950 (2014).

[15] G. Baur, Phys. Lett. B 178, 135 (1986).

[16] H.Esbensen, X.Tang, C.L.Jiang, Phys.Rev.C 84, 064613(2011).

[17] A. Diaz Torres, M. Wiescher, Phys.Rev.C 97,055802(2018)12.

[18] L.R. Gasques et al., Phys.Rev.C76,035802(2007).

[19] Le Hoang Chien et al., Phys. Rev. C 98, 064604 (2018).

[18] Dao T. Khoa et al., Nucl.Sci.Tech. 29,182(2018).

[19] K. Godbey, C. Simenel, A.S. Umar, Phys. Rev. C 100, 024619 (2019).

[20] M. Notani et al., Phys.Rev.C85,014607(2012).

[21] C.L. Jiang et al., Phys. Rev. Lett. 110, 072701 (2013).

[22] A. Bonasera and V.Kondratyev, Phys. Lett. B 339, 207(1994).

[23] V.N. Kondratyev, A. Bonasera and A.Iwamoto, PRC 61, 044613(2000).

[24] A. Bonasera and A. Iwamoto, Phys.Rev.Lett. 78, 187(1997).

[25] A. Bonasera, G. F. Bertsch, and E. El-Sayed, Phys. Lett. 141B, 9 (1984).

[26] A. Bonasera, Nucl. Phys. A439, 353(1985).

[27] A. Bonasera, Phys. Rev. C 34, 740(1986).

[28] R. Bass, Phys.Rev.Lett.39, 265(1977). 
[29] J. Randrup, Ann.Phys.(NY) 112, 356(1978).

[30] A.B. Balantekin, S.E. Koonin and J.W. Negele, Phys.Rev. C 28, 1656 (1983).

[31] S.Kimura and A.Bonasera, PRC 76, 031602(R)(2007).

[32] J. Ahrens et al., Nucl.Phys. A 251, 479(1975).

[33] G.Fruet et al., PRL124, 192701(2020).

[34] W.P. Tan et al., PRL124, 192702(2020).

[35] A.Bonasera and J.Natowitz, PRC 102, 061602(R)

(2020).

[36] T.Settlemyre et al., Nucl.Phys.A (2021) in press. 